\title{
Chip Technologies for Screening Chemical and Biological Agents Against Plant-Parasitic Nematodes
}

\author{
Augustine Q. Beeman, Zach L. Njus, Santosh Pandey, and Gregory L. Tylka
}

First and fourth authors: Department of Plant Pathology and Microbiology, and second and third authors: Department of Electrical and Computer Engineering, Iowa State University, Ames 50011.

Accepted for publication 14 July 2016.

\begin{abstract}
Beeman, A. Q., Njus, Z. L., Pandey, S., and Tylka, G. L. 2016. Chip technologies for screening of chemical and biological agents against plant-parasitic nematodes. Phytopathology 106:1563-1571.

Plant-parasitic nematodes cause substantial damage to agricultural crops worldwide. Long-term management of these pests requires novel strategies to reduce infection of host plants. Disruption of nematode chemotaxis to root systems has been proposed as a potential management approach, and novel assays are needed to test the chemotactic behavior of nematodes against a wide range of synthetic chemicals and root exudates. Two microfluidic chips were developed that measure the attraction or repulsion of nematodes to chemicals ("chemical chip") and young plant roots ("root chip"). The chip designs allowed for chemical concentration gradients to be maintained up to $24 \mathrm{~h}$, the nematodes to remain physically separate from the chemical reservoirs, and for images of nematode populations to be captured using either a microscope or a flatbed scanner. In the experiments

using the chemical chips, seven ionic solutions were tested on second-stage juveniles (J2s) of Meloidogyne incognita and Heterodera glycines. Results were consistent with previous reports of repellency of $M$. incognita to a majority of the ionic solutions, including $\mathrm{NH}_{4} \mathrm{NO}_{3}, \mathrm{KNO}_{3}, \mathrm{KCl}, \mathrm{MgCl}_{2}$, and $\mathrm{CaCl}_{2}$. H. glycines was found to be attracted to both $\mathrm{NH}_{4} \mathrm{NO}_{3}$ and $\mathrm{KNO}_{3}$, which has not been reported previously. A software program was written to aid in monitoring the location of nematodes at regular time intervals using the root chip. In experiments with the root chip, $H$. glycines $\mathrm{J} 2 \mathrm{~s}$ were attracted to roots of 3-day-old, susceptible (cultivar Williams 82) soybean seedlings, and attraction of $H$. glycines to susceptible soybean was similar across the length of the root. Attraction to resistant (cultivar Jack) soybean seedlings relative to the water only control was inconsistent across runs, and $H$. glycines J2s were not preferentially attracted to the roots of resistant or susceptible cultivars when both were placed on opposite sides of the same root chip. The chips developed allow for direct tests of plant-parasitic nematode chemotaxis to chemicals and roots with minimal human intervention.
\end{abstract}

The soybean cyst nematode, Heterodera glycines, and the rootknot nematode, Meloidogyne incognita, are economically important plant-parasitic nematodes, each causing billions of dollars in crop loss annually (Sasser and Freckman 1987). Plant-parasitic nematodes locate their hosts using a range of chemical cues exuded from host roots (Perry 1996). The selective movement of nematodes toward known and unknown chemical cues is referred to as chemotaxis (Zuckerman and Jansson 1984). In the soil, chemotaxis is a complex phenomenon where interplay among multiple attractants and repellents produce long-range and short-range spatiotemporal signals for the nematode (Reynolds et al. 2011). With limited energy reserves, nematodes need to decipher competing chemical cues and infect their host within a restricted time. Understanding the factors involved with plant-parasitic nematode chemotaxis is a scientifically intriguing topic that could lead to advances in managing these pests.

A number of assays have been developed and used to study the chemotaxis of nematodes. Many of these laboratory assays use agar as the migration matrix and use a number of different physical configurations (Castro et al. 1990; Devine and Jones 2003; Papademetriou and Bone 1983; Riddle and Bird 1985; Shinya et al. 2015). The configuration and dimensions of the areas used to conduct these assays depend on the scientific hypotheses being tested. For example, a Y-shaped olfactometer was created in a Pluronic gel

Corresponding author: G. L. Tylka; E-mail address: gltylka@iastate.edu

A. Q. Beeman and Z. L. Njus are joint first authors of this paper.

*The $\boldsymbol{e}$-Xtra logo stands for "electronic extra" and indicates that one supplementary figure is published online.

http://dx.doi.org/10.1094/PHYTO-06-16-0224-R

(c) 2016 The American Phytopathological Society matrix to study whether $M$. incognita and $M$. graminicola used the shortest path to navigate a maze and reach host roots (Reynolds et al. 2011). Besides the choice of the physical device, different ways to quantify the chemotactic behavior on plate assays have been reported. For instance, relative values can be assigned to nematodes depending on their proximity to a source compound (Wuyts et al. 2006), the number of nematodes in contact with a root (Wang et al. 2009 b), or by observing the tracks created by nematodes on agar surfaces as they move toward a filter paper disk soaked with a test compound (Huettel and Jaffe 1987; Papademetriou and Bone 1983). Although agar-based plate assays are widely adopted for their inherent simplicity, there are some compromises associated with their use. Plate assays require carefully drying the agar (Wuyts et al. 2006), and adding a treatment compound to a well cut into the agar or on a saturated filter paper disk (Hu et al. 2013; Papademetriou and Bone 1983), potentially introducing a source of variability between experiments. On plates with agar, some of the nematodes may not be visible throughout the length of the experiment as some nematodes can move beneath the agar surface, migrate to the edges of the plate, or become obscured as they reach the source of the chemical gradient (i.e., agar plug, filter paper disk, etc.). Recording tracks made by nematodes (Papademetriou and Bone 1983) to a test compound can also be challenging if there are many nematodes on a given plate.

As an alternative to agar-based plate assays, microfluidic technology offers the advantage of diffusing compounds within customdesigned chip geometries to suit a desired chemotaxis experiment. Microfluidics can be defined as "the handling and analyzing of fluids in structures of micrometer scale" (Beebe et al. 2002). In the past two decades, there have been exciting applications of microfluidics in biology, such as in electrophysiology, pharmaceutical drug screening, cell sorting and analysis, biomolecule separation and screening, point-of-care diagnostics, and tissue engineering (Beebe 
et al. 2002). Microfluidic systems may also be paired with technology to allow the user to automatically load samples, perfuse chemicals, monitor real-time activity, record and analyze data, and conduct multiple tests simultaneously and in parallel. In nematology, the development of microfluidic assays have been primarily focused on the behavioral modeling of Caenorhabditis elegans (Saldanha et al. 2013) and some parasites of veterinary importance (Chen et al. 2011). These studies have also used image analysis combined with microfluidics to aid in data acquisition (Njus et al. 2015; Saldanha et al. 2013).

While the goals of automation, real-time imaging, and highthroughput screening are necessary in certain studies (e.g., those involving pharmacology, toxicity, olfactory functions, and ionchannel kinetics of whole nematodes) that run over 10 or $20 \mathrm{~min}$ (Carr et al. 2011; Lockery et al. 2012; Peytavi et al. 2005), experiments to investigate the chemotaxis of plant-parasitic nematodes require longer time periods. Plant-parasitic nematodes exhibit a far more sedentary lifestyle than $C$. elegans, so microfluidic devices must be designed with these behavioral attributes in mind.

The objectives of our study were to (i) design, optimize, and fabricate two microfluidic chips, a "chemical chip" and "root chip"; (ii) determine the effect of multiple ionic solutions on movement of second-stage juveniles (J2s) of $H$. glycines and $M$. incognita using the chemical chip; and (iii) study the movement of H. glycines $\mathrm{J} 2 \mathrm{~s}$ in response to young soybean roots growing in the root chip. The combination of microfluidic chips and imaging tools can potentially lead to faster screening of novel chemical compounds that disrupt nematode migration to roots, characterizing nematode-resistant traits of host plants, and answering fundamental questions in root-nematode interactions.

\section{MATERIALS AND METHODS}

Microfluidic chips. The overall design of the chemical microfluidic chip is shown in Figure 1. Each lane on the chip consisted of a central port (i.e., the nematode entry port) where nematodes were inserted for chemotaxis experiments (Fig. 1A). Two resting chambers on either side of the nematode entry port allowed for unrestricted movement of the nematodes from the central port. Two resting chambers at opposite ends of the lane provided access to reservoirs that contained treatment solutions. The treatment reservoir housed the test compound of interest and the control reservoir contained the experimental control treatment. The resting chambers were connected to the treatment and control reservoirs by microscale filters (Fig. 1B). The filters allowed slow diffusion of chemicals from the treatment and control reservoirs into the resting chambers and also prevented nematodes from moving into the treatment and control reservoirs. The chemical microfluidic chip contained four parallel lanes for separate, simultaneous tests (Fig. 1C). The dimensions of all components of the chip are included in Supplementary Figure S1.

The mask templates were drawn in AutoCAD and sent to an outside vendor (FineLine Imaging) for printing. The microfluidic chip mold was made in two parts: first the filters were made, followed by the chambers and ports. Standard soft lithography was used to fabricate the microfluidic chip. Briefly, an ultravioletsensitive polymer, SU-8 2005 (MicroChem), was spin coated at a speed of 3,000 rpm for $30 \mathrm{~s}$ on a $7.62 \mathrm{~cm}$ silicon wafer to deposit a 5 - $\mu$ m-thick layer. The wafer then was baked at $95^{\circ} \mathrm{C}$ for $2 \mathrm{~min}$. The SU-8 layer was selectively exposed to ultraviolet light $(350 \mathrm{~nm})$ using the mask template for the filters. The wafer then was baked for $3 \mathrm{~min}$ at $95^{\circ} \mathrm{C}$ to harden the developed SU-8. A second layer of photoresist, SU-8 2025 (MicroChem), was spin coated on top of the 5 - $\mu \mathrm{m}$-thick layer of SU-8 for $30 \mathrm{~s}$ at $1,000 \mathrm{rpm}$ resulting in a layer thickness of $75 \mu \mathrm{m}$. Next, the wafer was baked at $65^{\circ} \mathrm{C}$ for $3 \mathrm{~min}$ and then baked for an additional $9 \mathrm{~min}$ at $95^{\circ} \mathrm{C}$. The mask template for the ports and chambers then was used to selectively expose the SU-8 to the ultraviolet light. After another baking step at $65^{\circ} \mathrm{C}$ for $2 \mathrm{~min}$ and $95^{\circ} \mathrm{C}$ for $7 \mathrm{~min}$, a SU-8 developer (MicroChem) was used to remove the unexposed photoresist leaving a negative mold of the final chip. Then to fully harden the SU-8, the wafer was baked at $150^{\circ} \mathrm{C}$ for $30 \mathrm{~min}$. Thereafter, polydimethylsiloxane (PDMS) was poured on the SU-8 mold and baked on a hotplate for $3 \mathrm{~h}$ at $100^{\circ} \mathrm{C}$. The solidified PDMS structure was peeled off and holes were punched to create the treatment and control reservoirs and the

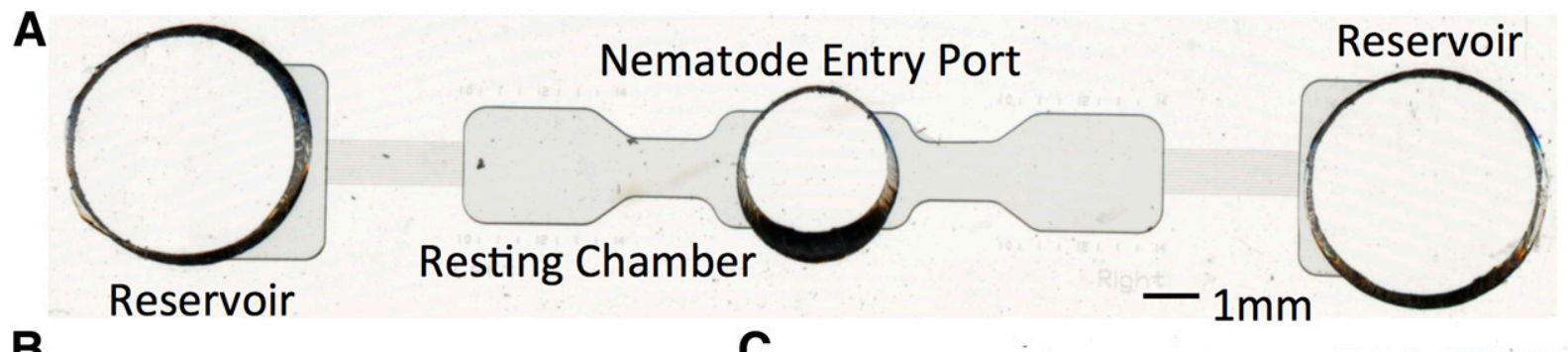

B
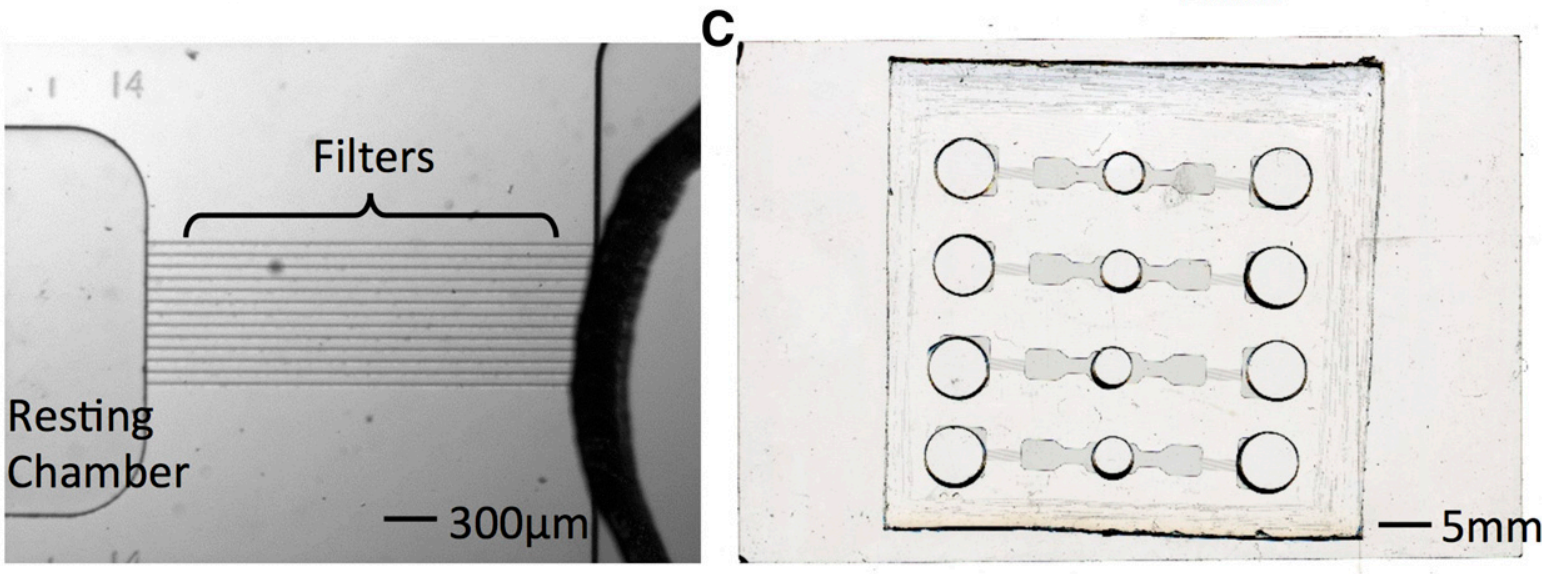

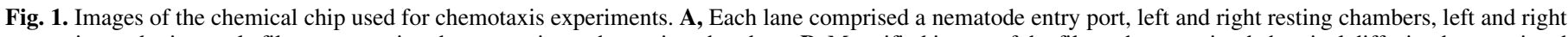

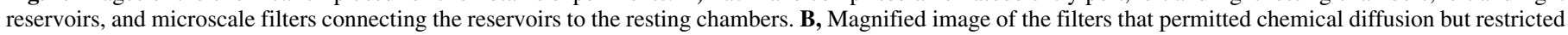

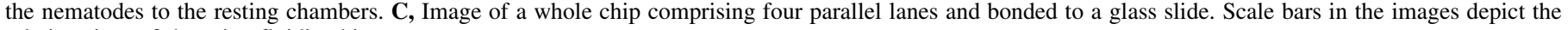
relative sizes of the microfluidic chip. 
nematode entry port. Finally, the PDMS chip was bonded to a glass slide using a plasma cleaner. Preliminary experiments were conducted with $H$. glycines $\mathrm{J} 2$ s to determine the optimal device layout and dimensions, particularly the size of the filters and the relative volumes of resting chambers and reservoirs for use with plant-parasitic nematodes.

Figure 2A shows diffusion tests with colored dyes taken at different time points to confirm the slow diffusion of chemicals into the resting chambers over the length of an experiment. A microfluidic lane in the chip was prefilled with water, and red and blue dyes were added to the treatment and control reservoirs. As shown in the figure, the two dyes are localized in their respective resting chambers, and there is virtually no mixing of the dyes in the central nematode entry port. This visual observation suggests that the microfluidic chips can be used to perform chemotaxis experiments for up to $24 \mathrm{~h}$. Figure $2 \mathrm{~B}$ demonstrates how nematodes move through the chemical chip over the length of an experiment with $\mathrm{KNO}_{3}$. The H. glycines $\mathrm{J} 2 \mathrm{~s}$ in this run were attracted to $\mathrm{KNO}_{3}$ as early as $4 \mathrm{~h}$, and a majority of the nematodes in the chip were in the resting chamber nearest to the filters connected to the $\mathrm{KNO}_{3}$ reservoir by $24 \mathrm{~h}$.

The chemical chip was modified into a root chip by converting the four separate treatment and control reservoirs in the four lanes of the chip into two elongated root channels. This was done by carefully cutting a PDMS layer with a clean razor blade and bonding two rectangular pieces of PDMS over the top of the root channels. This design provided coverage of the root and slowed the evaporation of fluids from the root channel. The root chip comprised four lanes that are exposed to different sections of the growing root, from nearest the seed to nearest the root tip.

Nematode collection for experiments. Populations of $H$. glycines and $M$. incognita were maintained in the greenhouse on soybean, Glycine max (cultivar Williams 82) and tomato, Lycopersicon esculentum (cultivar Rutgers), respectively. Females and cysts (dead females filled with eggs) of $H$. glycines were collected from 4- to 8-week-old plants by rinsing roots with water to remove soil and then spraying the roots with a stream of water on an $850-\mu \mathrm{m}$ pore sieve nested above a $250-\mu \mathrm{m}$-pore sieve. The debris and the $H$. glycines females and cysts were collected on the $250-\mu \mathrm{m}$-pore sieve, and the females and cysts were separated from the debris by sucrose centrifugation (Jenkins 1964) using sucrose at 1,362 g/liter. The females and cysts were crushed with a motorized rubber stopper to release the eggs, which were collected on a $25-\mu \mathrm{m}$-pore sieve nested under a 75- $\mu$ m-pore sieve (Faghihi and Ferris 2000).

Eggs of $M$. incognita were collected by washing soil from the roots of 6- to 8-week-old $M$. incognita-infected tomato plants, cutting the roots into 2 -cm-long pieces, and incubating the root pieces with agitation in $0.5 \%$ sodium hypochlorite (bleach) for 2 min (Hussey and Barker 1973). The suspension was poured over a nested pair of $75-\mu \mathrm{m}$-pore and $25-\mu \mathrm{m}$-pore sieves to recover the nematode eggs on the bottom, $25-\mu \mathrm{m}$-pore sieve. The eggs were rinsed with water several times to remove all residues of the bleach.

Extracted eggs of both nematode species were further separated from debris with sucrose centrifugation in sucrose at $454 \mathrm{~g} / \mathrm{liter}$ (Jenkins 1964). The eggs were washed with sterile, distilled water and incubated in the dark at $25^{\circ} \mathrm{C}$ for $72 \mathrm{~h}$ on $30-\mu \mathrm{m}$-pore mesh (Elko Filtering, Miami, FL) to hatch the J2s (Wong et al. 1993). The hatched J2s moved downward through the $30-\mu \mathrm{m}$-pore mesh into the water and were collected by centrifuging the nematode suspension at 2,000 $\times g$ for approximately $4 \mathrm{~min}$. The concentrated $\mathrm{J} 2$ suspension was diluted with sterile distilled water to achieve the appropriate nematode population density for use in the experiments.

Chemical chip experimental setup. Each lane of the microfluidic chip was filled with approximately $100 \mu \mathrm{l}$ of sterile distilled water added through the nematode entry port using a 100-ml syringe connected to rubber tubing (inner diameter: $1 \mathrm{~mm}$ ). A custom-made PDMS connector was put over the nematode entry port to apply sufficient fluid pressure to completely fill the chip. The connector was a cylindrical piece of PDMS (outside diameter: $5 \mathrm{~mm}$, height $3 \mathrm{~mm}$ ) with a $0.8-\mathrm{mm}$-diameter hole punch in the center, which allowed the tubing to be attached the connector. The chips were visually inspected after filling to ensure that each entire lane was completely filled with water. If air bubbles were trapped in any section of the lane, more water was added until the bubbles had exited the lane through openings of the treatment or control reservoirs. Chips that had large bubbles in the resting chambers or in the filters that could not be removed were discarded. Next, 30 to 70 $H$. glycines or $M$. incognita $\mathrm{J} 2 \mathrm{~s}$ were suspended in 10 to $15 \mu \mathrm{l}$ of water and added to the nematode entry port, and then $50 \mu \mathrm{l}$ of test chemical was added to the treatment reservoir and $50 \mu \mathrm{l}$ of water was added to the control reservoir. The compounds used in the experiments were ionic solutions that had been reported to be attractants or repellants for $H$. glycines (Papademetriou and Bone 1983) and M. incognita (Castro et al. 1990; Le Saux and Quénéhervé 2002; Prot 1979; Riddle and Bird 1985). These included sulfates $\left(\mathrm{Na}_{2} \mathrm{SO}_{4}, \mathrm{ZnSO}_{4}\right)$, nitrates $\left(\mathrm{NH}_{4} \mathrm{NO}_{3}, \mathrm{KNO}_{3}\right)$, and chlorides $\left(\mathrm{MgCl}_{2}\right.$, $\mathrm{CaCl}_{2}, \mathrm{KCl}$ ). All ionic solutions were prepared at a concentration of $500 \mathrm{mM}$. Ionic solution treatments were added to the left or right sides arbitrarily, and their position was switched for each replication. To check for any bias of nematode movement from other factors (such as

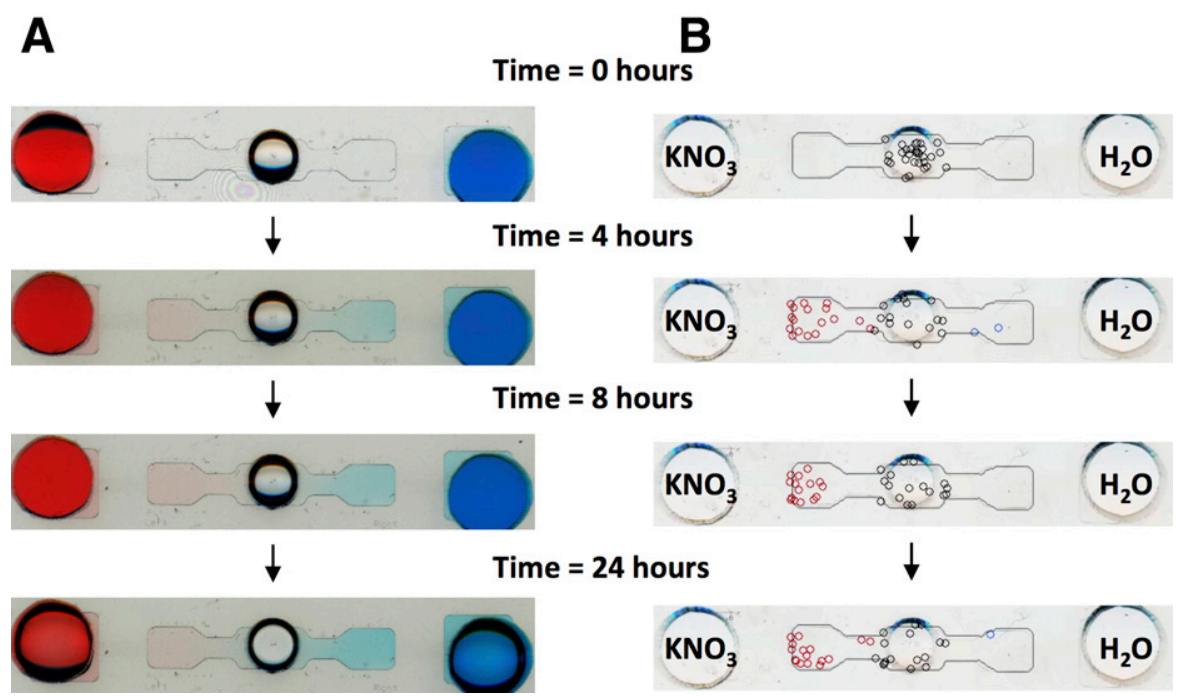

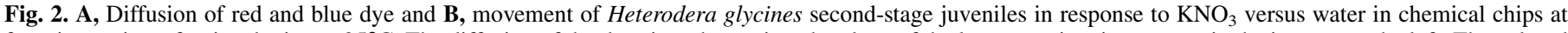

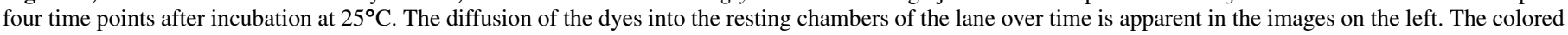

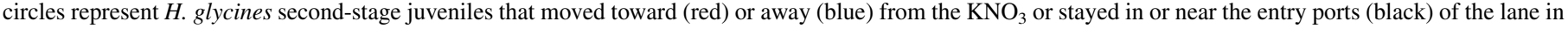
the microfluidic chip in the images on the right. 
chip dimensions, fluid flow, or filling process), some lanes were filled with water in both the treatment and control reservoirs. The chips were incubated in darkness at $25^{\circ} \mathrm{C}$. For each ionic solution, between 482 to $712 \mathrm{H}$. glycines J2s were tested over a total of 9 to 10 replications and 441 to $666 \mathrm{~J} 2 \mathrm{~s}$ of $M$. incognita were tested over a total of 9 to 12 replications (Table 1 ).

Root chip experimental setup. Experiments were conducted with $H$. glycines and soybean seedlings in root chips. The following set of treatments were assessed: $H$. glycines-susceptible soybean versus water, $H$. glycines-resistant soybean versus water, $H$. glycinessusceptible soybean versus $H$. glycines-resistant soybean, and water versus water. Soybean seeds of $H$. glycines-susceptible (Williams 82) and $H$. glycines-resistant (Jack) cultivars were plated on $1.5 \%$ agar and germinated in darkness at $25^{\circ} \mathrm{C}$ for 3 days. For testing chemotaxis of $\mathrm{H}$. glycines to single cultivars, roots of these seedlings were inserted arbitrarily in a channel on either side of the root chip, and the other channel was filled with water. In a separate experiment, roots of the two soybean cultivars were placed on opposite sides of a chip. Similar to the chemical chip setup, water-only controls were used to check for any directional bias in the chips in which the root channels were filled with water on both sides. The $H$. glycines $\mathrm{J} 2 \mathrm{~s}$ were added to the nematode entry port just as they were for the chemical chip experiments, and the movement of the $\mathrm{J} 2 \mathrm{~s}$ toward or away from these treatments was monitored. Root chips were placed flat on a scanner that was enclosed in the temperature-controlled $\left(25^{\circ} \mathrm{C}\right)$ chamber and scanned every hour for $18 \mathrm{~h}$. A total of 4 chips were used for each treatment, with the number of H. glycines $\mathbf{J} 2$ s tested for each treatment ranging from 502 to 574 .

Data collection. For the chemical chip experiments, all data were collected using a dissecting microscope at $60 \times$ magnification. The locations of the nematodes in the chemical chips were initially recorded at $1,6,24$, and $48 \mathrm{~h}$ after the start of the experiment. It was then determined that the maximum response occurred at $24 \mathrm{~h}$, and data collection for some chemical chip experiments was done only at $24 \mathrm{~h}$.

For experiments with the root chip, a custom software program was written in AutoHotkey to control a high-resolution (2,400 dpi) flatbed scanner (EPSON Perfection V750-M Pro Scanner) onto which the root chips were placed. The program also controlled the time interval between successive scans of the chips. A temperaturecontrolled chamber $\left(25^{\circ} \mathrm{C}\right)$ housed the scanner, and multiple chips (up to four) were placed on the scanner bed to be monitored

TABLE 1. Effect of different ionic solutions $(500 \mathrm{mM})$ on chemotaxis of two plant-parasitic nematodes ${ }^{\mathrm{a}}$

\begin{tabular}{|c|c|c|c|c|c|}
\hline Ionic solution & Replicates & $\begin{array}{c}\text { Total } \\
\text { nematodes }\end{array}$ & Outcome & $P$ value & $\begin{array}{c}\text { Standard } \\
\text { error }\end{array}$ \\
\hline \multicolumn{6}{|l|}{$\begin{array}{r}\text { Meloidogyne } \\
\text { incognita }\end{array}$} \\
\hline $\mathrm{CaCl}_{2}$ & 12 & 626 & $\mathrm{R}$ & 0.0007 & 7.79 \\
\hline $\mathrm{MgCl}_{2}$ & 10 & 496 & $\mathrm{R}$ & 0.0004 & 9.05 \\
\hline $\mathrm{KCl}$ & 11 & 441 & $\mathrm{R}$ & $<0.0001$ & 6.08 \\
\hline $\mathrm{NH}_{4} \mathrm{NO}_{3}$ & 12 & 768 & $\mathrm{R}$ & $<0.0001$ & 6.63 \\
\hline $\mathrm{KNO}_{3}$ & 11 & 666 & $\mathrm{R}$ & 0.0002 & 7.36 \\
\hline $\mathrm{ZnSO}_{4}$ & 10 & 515 & $\mathrm{~N}$ & 0.1798 & 6.28 \\
\hline $\mathrm{Na}_{2} \mathrm{SO}_{4}$ & 9 & 555 & $\mathrm{~N}$ & 0.2435 & 4.46 \\
\hline Water & 23 & 1363 & $\mathrm{~N}$ & 0.9185 & 5.26 \\
\hline \multicolumn{6}{|l|}{$\begin{array}{l}\text { Heterodera } \\
\text { glycines }\end{array}$} \\
\hline $\mathrm{CaCl}_{2}$ & 10 & 690 & $\mathrm{R}$ & 0.0332 & 7.42 \\
\hline $\mathrm{MgCl}_{2}$ & 10 & 483 & A & 0.0195 & 7.79 \\
\hline $\mathrm{KCl}$ & 10 & 627 & $\mathrm{~N}$ & 0.6436 & 9.52 \\
\hline $\mathrm{NH}_{4} \mathrm{NO}_{3}$ & 9 & 473 & A & 0.0058 & 5.83 \\
\hline $\mathrm{KNO}_{3}$ & 10 & 712 & A & $<0.0001$ & 6.20 \\
\hline $\mathrm{ZnSO}_{4}$ & 9 & 561 & $\mathrm{~N}$ & 0.5131 & 0.05 \\
\hline $\mathrm{Na}_{2} \mathrm{SO}_{4}$ & 9 & 546 & $\mathrm{~N}$ & 0.2157 & 0.04 \\
\hline Water & 23 & 1458 & $\mathrm{~N}$ & 0.1634 & 0.06 \\
\hline
\end{tabular}

a Results of chemotaxis experiments were taken at $24 \mathrm{~h}$. A total of 9 to 12 replications were done for each ionic solution tested. For each species of nematode, the response was rated as either $\mathrm{A}=$ attracted, $\mathrm{N}=$ neutral response, or $\mathrm{R}=$ repelled at the $P=0.05$ level using a Student's paired $t$ test. simultaneously during an experiment. When the experimental run was completed, a second program written in MATLAB organized and formatted the scanned images to allow a user to identify the locations of all of the nematodes in a lane at each time point. Thereafter, the MATLAB program used the $\mathrm{x}$ - and $\mathrm{y}$-coordinates of each user-identified nematode and scored individual nematodes as either being attracted, repelled, or having no preference to the treatment based on their location in the resting chambers or nematode entry port. The movement of $\mathrm{J} 2 \mathrm{~s}$ to a resting chamber adjacent to a reservoir holding a test compound was considered an attraction behavior, and movement of $\mathrm{J} 2 \mathrm{~s}$ to a resting chamber in the opposite direction was considered a repulsion behavior. We also used the scanner setup to demonstrate the slow diffusion of chemicals through the filters from the treatment ports (Fig. 2).

Statistical analysis. For each nematode species in the chemical chip experiments, there were between 9 to 12 runs for each ionic solution tested and 23 runs for the water only control treatment. The percentages of $\mathrm{J} 2 \mathrm{~s}$ in the two resting chambers and nematode entry port at $24 \mathrm{~h}$ were calculated. For the chemical chip experiments, the percentage of nematodes in a resting chamber adjacent to the treatment reservoir was compared with the percentage of nematodes in the resting chamber adjacent to the control reservoir by a paired Student's $t$ test in SAS using PROC TTEST (Devine and Jones 2003; Hu et al. 2013).

For the root chip experiments, the percentages of nematodes in each of the resting chambers were analyzed similarly to the chemical chip experiments. First, an analysis of variance was conducted in PROC GLM of SAS (version 9.4) to test the effect of lane at each hour time point. If the effect of lane was found not to be significant, the data from all lanes were combined for analysis and the effect of each treatment in the root chips at $18 \mathrm{~h}$ was tested using a paired $t$ test in PROC TTEST.

A chemotaxis index (chemoindex) modeled after Le Saux and Quénéhervé (2002) was calculated to determine overall movement of the nematode population inside each lane of the root chip. The equation for the chemoindex was defined as $\mathrm{pT}-\mathrm{pC}$, where $\mathrm{pT}$ is the percentage of nematodes in the chip residing in the resting chamber adjacent to the treatment reservoir and $\mathrm{pC}$ is the percentage of the nematodes in the resting chamber adjacent to the control reservoir. Therefore, a positive value (i.e., greater than zero) denoted that the nematode juveniles were attracted to the treatment, and a negative value (i.e., less than zero) indicated that juveniles were repelled from the treatment. The chemoindex value was calculated for each lane of a chip at every time point in the experiment. Standard errors of the mean of the chemoindex values for each lane were calculated and plotted for every hour of the experiment.

\section{RESULTS}

Chemical chip experiments. Greatest separation of both plantparasitic nematode species from the resting chambers occurred by $24 \mathrm{~h}$ of incubation (data from other time points are not shown). No directional bias toward the right or left side of the chip was observed in the water only controls (Fig. 3). H. glycines and M. incognita exhibited differential chemotactic responses to the ionic solutions. In general, 32 to $52.3 \%$ of the $H$. glycines $\mathrm{J} 2 \mathrm{~s}$ added to the chemical chips moved toward or away from test compounds and 37.6 to $76.6 \%$ remained in the nematode entry point after $24 \mathrm{~h}$. In contrast, only 14.1 to $51 \%$ of the $M$. incognita $\mathrm{J} 2$ s moved toward or away from compounds used in the experiments.

A compound was determined to be an attractant or repellent if a significantly higher proportion of nematodes moved toward the nematode resting chamber adjacent to treatment reservoir or away from a compound being tested (i.e., toward the resting chamber adjacent to the control reservoir). Compounds that elicited no significant movement toward or away from the source were considered neutral. Among the compounds to which H. glycines was exposed, three were attractants, one was a repellant, and three 
had no significant effect (Table 1). Specifically, there was significant movement of $\mathrm{H}$. glycines $\mathrm{J} 2$ s toward $\mathrm{MgCl}_{2}, \mathrm{KNO}_{3}$, and $\mathrm{NH}_{4} \mathrm{NO}_{3}$ (Fig. 3). H. glycines J2s were most strongly attracted to $\mathrm{KNO}_{3}$, where an average of $51 \%$ of the $\mathrm{J} 2 \mathrm{~s}$ in the chip moved toward the compound and only $10.1 \%$ away. Conversely, H. glycines was repelled by $\mathrm{CaCl}_{2}$, with an average of $13.2 \%$ moving toward the compound and $32 \%$ moving away. There was no significant movement detected toward or away from $\mathrm{KCl}, \mathrm{ZnSO}_{4}$, $\mathrm{Na}_{2} \mathrm{SO}_{4}$, and the water only control. In contrast to the results obtained with $H$. glycines, $M$. incognita $\mathrm{J} 2 \mathrm{~s}$ were repelled by $\mathrm{KNO}_{3}$, $\mathrm{NH}_{4} \mathrm{NO}_{3}, \mathrm{KCl}, \mathrm{MgCl}_{2}$, and $\mathrm{CaCl}_{2}$. For example, an average of $51 \%$ of the $M$. incognita $\mathrm{J} 2$ s move away by $\mathrm{KCl}$ and only $1.3 \%$ moved toward the compound (Fig. 3). Like H. glycines, M. incognita J2s were not attracted or repelled by $\mathrm{ZnSO}_{4}$ and $\mathrm{Na}_{2} \mathrm{SO}_{4}$ (Table 1).

Root chip experiment. These experiments were limited to $18 \mathrm{~h}$, largely because water uptake from roots resulted in empty root channels after this time. The $H$. glycines $\mathrm{J} 2 \mathrm{~s}$ were successfully tracked moving in the chip during the course of the experiment using a flatbed scanner enclosed in a temperature-controlled box (Fig. 4). For the treatments with roots, the lane positions had negligible effect on the nematode movement patterns, and data were subsequently combined and analyzed (Table 2). The

M. incognita

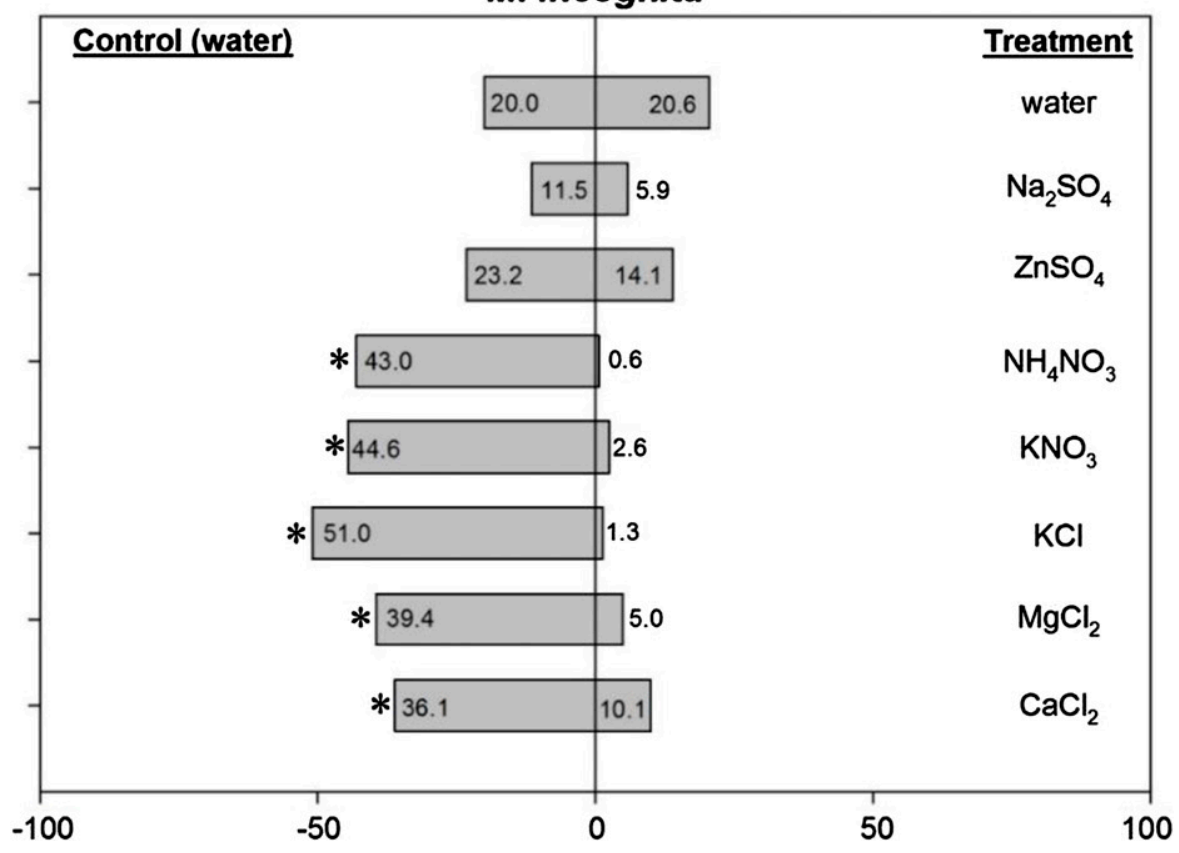

H. glycines

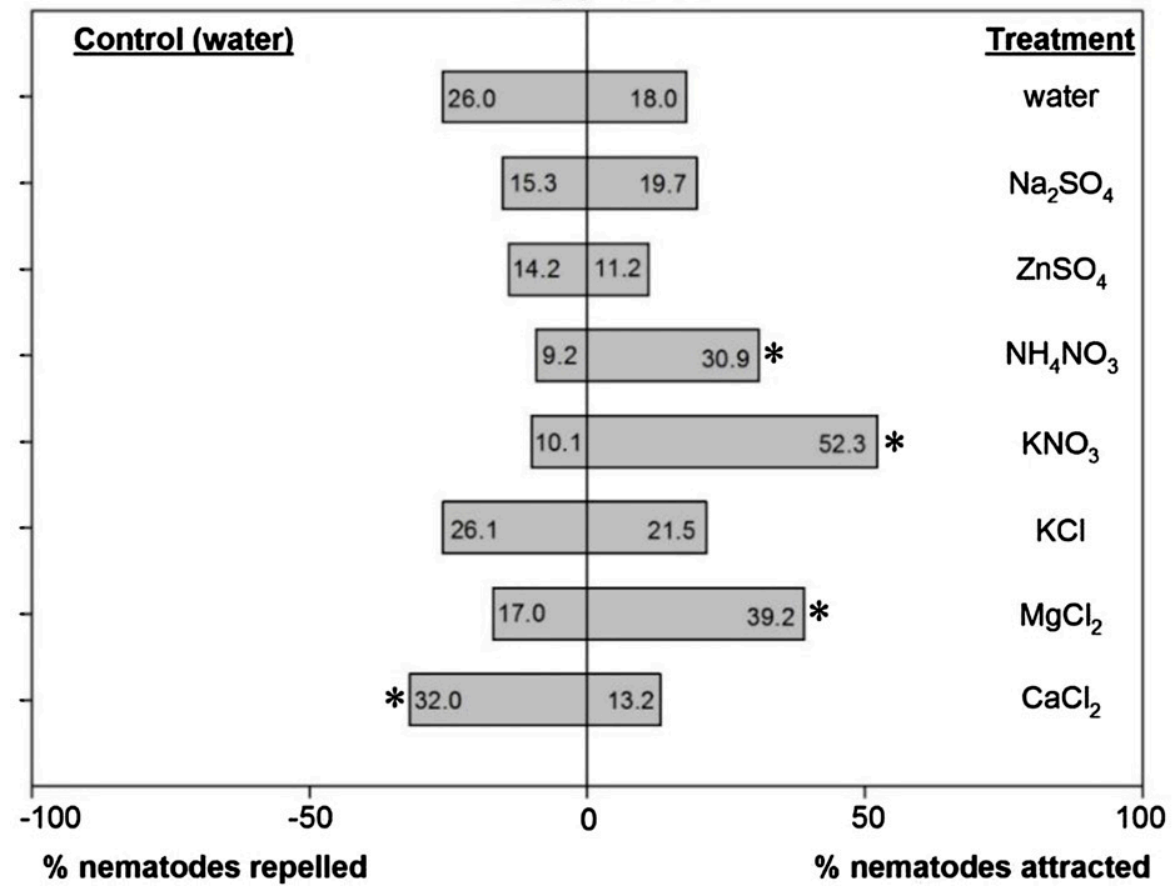

Fig. 3. Results after $24 \mathrm{~h}$ from the chemical chip chemotaxis experiments using different ionic solutions (500 mM) on juveniles of Meloidogyne incognita and Heterodera glycines. Bars represent the percentage of total nematodes in each lane moving either toward or away from ionic solutions. Each bar represents pooled data from all runs ( $n=9$ to 23; Table 1). Asterisks indicate a significant difference between the percentage of attracted and repelled juveniles at $P=0.05$ using a Student's paired $t$ test. 
$H$. glycines-susceptible cultivar attracted $H$. glycines $\mathrm{J} 2$ s regardless of which lane the nematodes were placed in, with positive chemotaxis index values ranging by lane from 12.1 to 16.8 after $18 \mathrm{~h}$ (Fig. 5A). When lanes were combined for analysis, this attraction to the susceptible root was found to be significant (Table 2). H. glycines J2s were attracted to $H$. glycines-resistant soybean in some runs, but the attractive response was not consistently seen across all replications, with chemotaxis index values ranging by lane from -9.2 to 3.5 after $18 \mathrm{~h}$ (Fig. 5B). There was no strong preference to either root when roots from both $H$. glycines-resistant and $H$. glycinessusceptible cultivars were present on opposite sides (Fig. 5C), with most nematodes remaining in the central port at the end of the experiment. There was no directional bias in the water-only control chips (Fig. 5D).

\section{DISCUSSION}

Plant-parasitic nematodes use chemical signals in the soil to guide them to host roots. This host-finding behavior provides scientists with an opportunity to search for chemical compounds and biological agents that disrupt obligately parasitic nematodes from finding host roots. Known chemical signals to which nematodes respond include plant hormones, $\mathrm{CO}_{2}$, ionic gradients, $\mathrm{pH}$ gradients, and secondary metabolites (Curtis 2008;
Dusenbery 1983; Kerry 2000; Riddle and Bird 1985; Wang et al. 2009a; Wuyts et al. 2006). Root exudates likely contain components that both attract and repel nematodes (Devine and Jones 2003; Tefft and Bone 1985). Some scientists have tried to characterize the effects of root exudates on nematodes (Devine and Jones 2003), but more studies are needed to understand the role of various factors in nematode chemotaxis. A better understanding of nematode chemotaxis can lead to identifying compounds that could serve as crop protection chemicals (Le Saux and Quénéhervé 2002) or biological antagonists (Hu et al. 2013; Sikora 1992). Host plant resistance has been reported based partially on reduced nematode attraction to roots (Linsell et al. 2014), and exudates from root-cap cells have been reported to induce quiescence in certain plant-parasitic nematodes (Hiltpold et al. 2014). Furthermore, production of peptides in planta that disrupt chemotaxis of cyst nematodes has been reported (Liu et al. 2005). The microfluidic chips described in this paper can aid in conducting research to develop novel management strategies.

In this paper, we describe two microfluidic chips to study the chemotaxis of plant-parasitic nematodes to both chemical compounds and live roots of intact soybean seedlings. The designs incorporate microscale filters that physically separate the nematodes from the treatments while permitting the establishment of chemical gradients over a period of 18 to $24 \mathrm{~h}$. The chips enable increased standardization in chemotaxis experiments because the

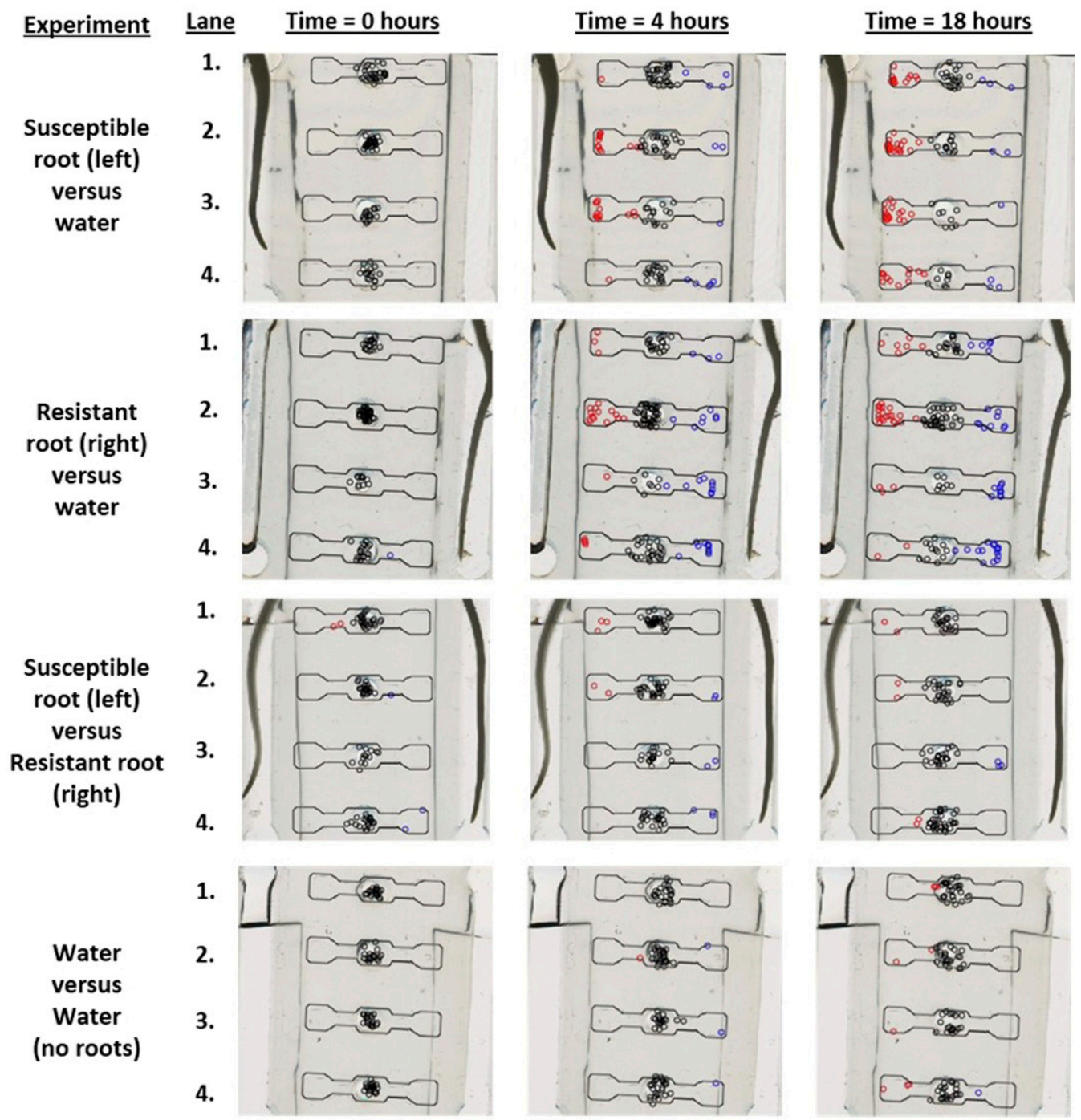

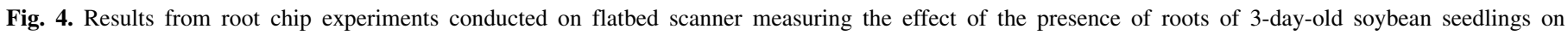

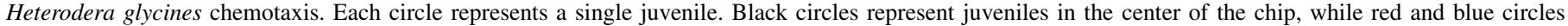
represent juveniles that have migrated to the left and right portions of the chip, respectively. 
mask template is reusable, allowing us to fabricate new batches of microfluidic chips with consistent repeatability in chip dimensions. Additionally, we developed a software program that helps to analyze a large set of saved images-identifying moving nematodes and tracking the movement of nematode populations as a function of time. Seven ionic solutions were tested on chemotaxis of $M$. incognita and $H$. glycines $\mathrm{J} 2 \mathrm{~s}$ using visual observation of large numbers of nematodes ( $>5,000$ of each species) with the chemical chip. And we measured the effect of soybean roots on chemotaxis of $H$. glycines with the root chip using a flatbed scanner paired with software to identify the position of all the nematodes in a device at $1 \mathrm{~h}$ time points over the course of an $18 \mathrm{~h}$ experiment.

The results of the experiments with the chemical chip revealed an interesting differential chemotactic response of $M$. incognita and $H$. glycines $\mathrm{J} 2 \mathrm{~s}$ to various ionic solutions. M. incognita $\mathrm{J} 2 \mathrm{~s}$ were significantly repelled by all but two ionic solutions tested, which was consistent with results from previous studies reporting strong repellency of $M$. incognita to various ionic solutions (Castro et al. 1990; Le Saux and Quénéhervé 2002; Prot 1979; Riddle and Bird 1985). The mechanism behind this repellent behavior of $M$. incognita J2s to ionic solutions has not been discerned, but recent research by Hida et al. (2015) indicates that $\mathrm{KNO}_{3}$ can be both an attractant and repellent at different concentrations. Our research did not examine concentration-dependent effects.

Chemotaxis of $H$. glycines $\mathbf{J} 2$ s has been less studied than that of M. incognita, but Papademetriou and Bone (1983) found that $\mathrm{ZnSO}_{4}$ and $\mathrm{MgCl}_{2}$ attracted $H$. glycines $\mathrm{J} 2 \mathrm{~s}$. In our experiments, $\mathrm{MgCl}_{2}$ attracted $H$. glycines $\mathrm{J} 2 \mathrm{~s}$ but $\mathrm{ZnSO}_{4}$ did not elicit an attractive or repellent response. Differences between our results and those reported by the literature can possibly be attributed to the

TABLE 2. Effect of 3-day-old Heterodera glycines-susceptible (Williams 82) and H. glycines-resistant (Jack) soybean roots on chemotaxis of $H$. glycines juveniles $^{\mathrm{a}}$

\begin{tabular}{|c|c|c|c|c|c|c|c|}
\hline Treatment & Control & Replicates (all lanes) & Total nematodes & $\begin{array}{l}P \text { value for } \\
\text { effect of lane }\end{array}$ & $\begin{array}{c}\text { Outcome } \\
\text { (combined lanes) }\end{array}$ & $\begin{array}{l}P \text { value for } \\
\text { paired } t \text { test }\end{array}$ & $\begin{array}{c}\text { Standard } \\
\text { error }\end{array}$ \\
\hline Susceptible & Water & 16 & 502 & 0.8499 & A & 0.001 & 3.70 \\
\hline Resistant & Water & 16 & 573 & 0.4183 & $\mathrm{~N}$ & 0.90 & 3.79 \\
\hline Susceptible & Resistant & 16 & 576 & 0.9845 & $\mathrm{~N}$ & 0.21 & 5.81 \\
\hline Water & Water & 16 & 524 & 0.9253 & $\mathrm{~N}$ & 0.11 & 2.33 \\
\hline
\end{tabular}

${ }^{a}$ Results are for root chip experiments at $18 \mathrm{~h}$. Effect of lane was determined by analysis of variance at $18 \mathrm{~h}$ for each of the treatments. Data from the percentage of juveniles migrating to each side of the chip was then analyzed, and the response was rated as either $\mathrm{A}=$ attracted, $\mathrm{N}=$ neutral response, or $\mathrm{R}=$ repelled at the $P=0.05$ level using a Student's paired $t$ test.

A
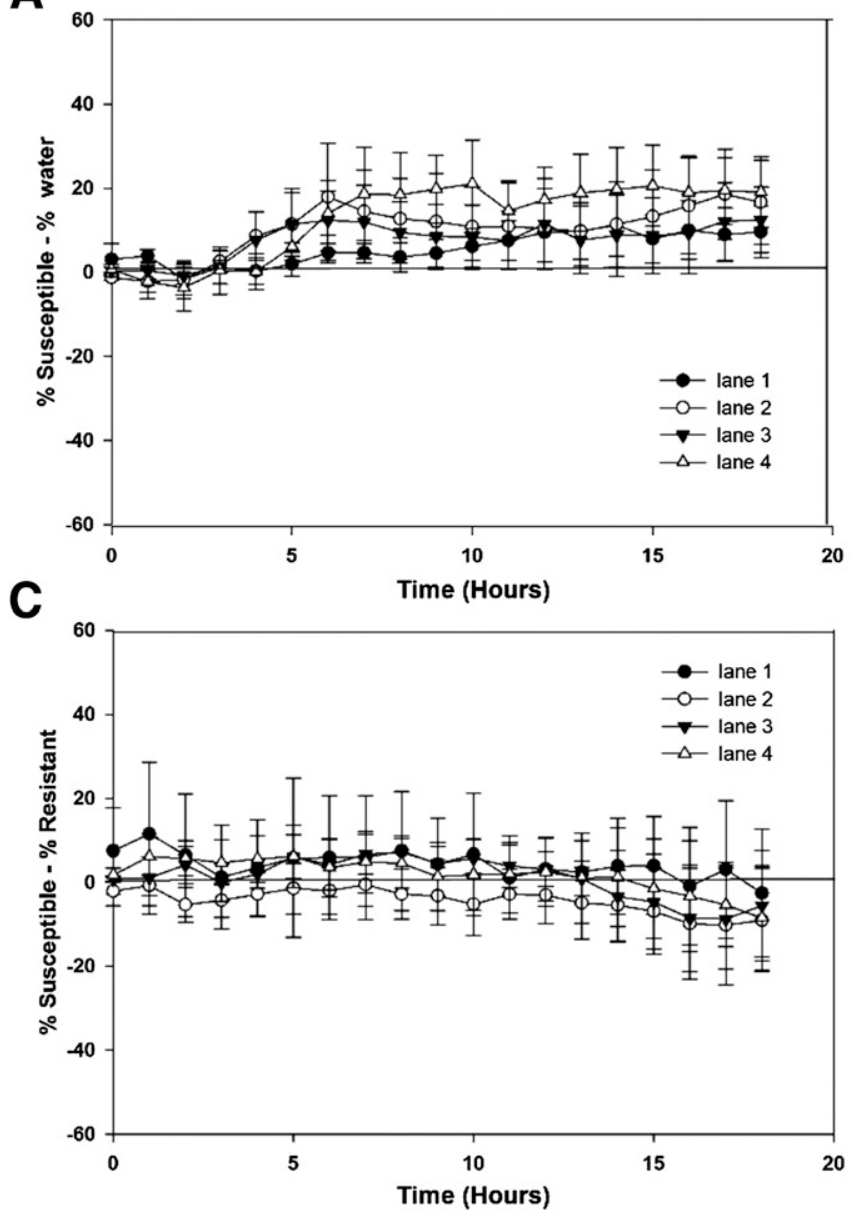

B
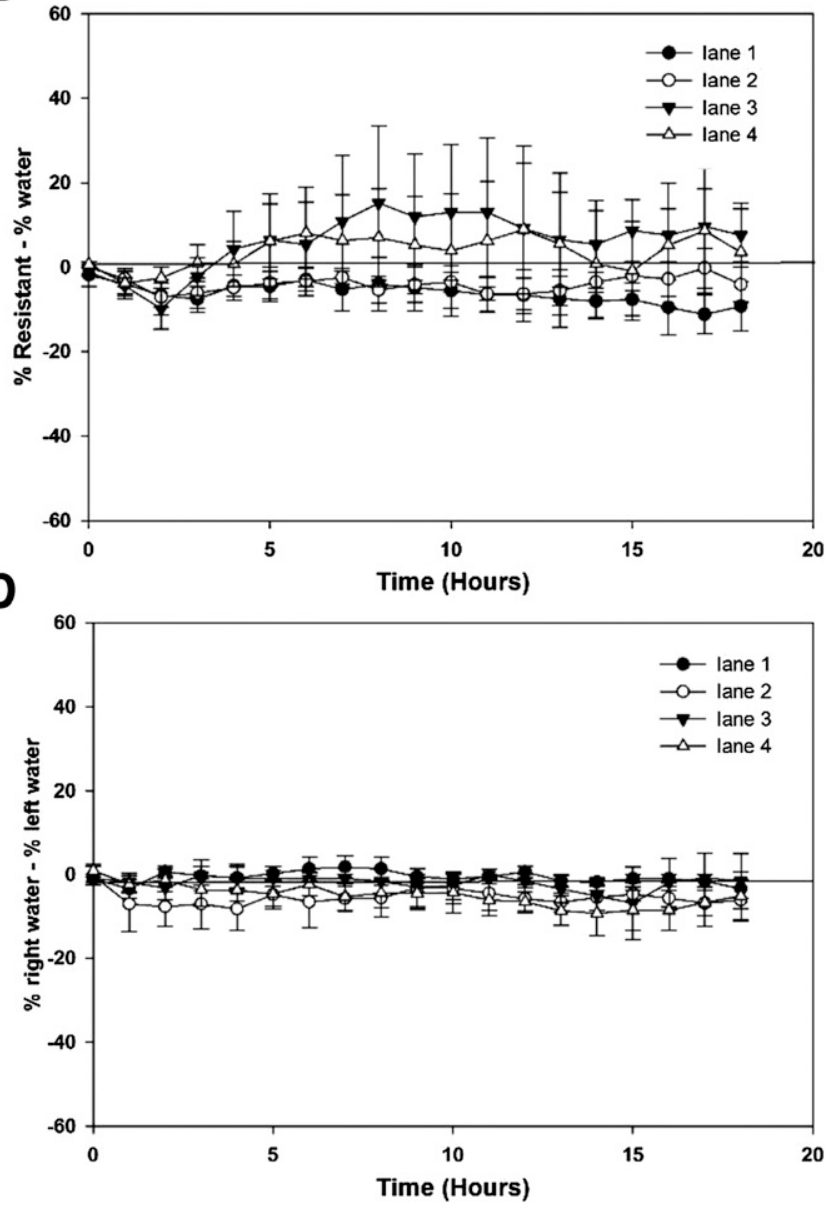

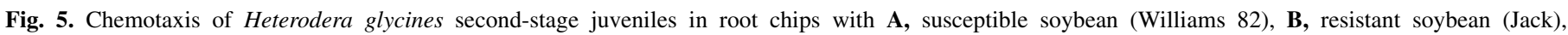

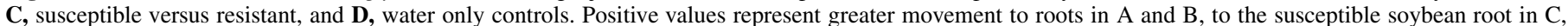

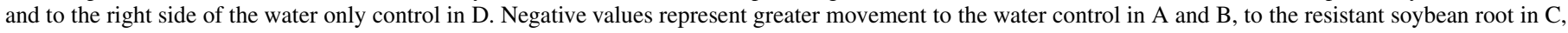
and to the left side of the water only control in D. Error bars represent the standard error of the mean for each lane-hour combination. 
difference in chemical diffusion in different incubation media (water, agar, Pluronic gel, etc.) or the layout of the assay. For example, the assay of Papademetriou and Bone (1983) measured attractive behavior indirectly based on agar tracks as an indication to whether the nematodes moved toward filter paper saturated with a test compound over a $24 \mathrm{~h}$ period and $\mathrm{ZnSO}_{4}$ was found to be an attractant. Our chip allows for direct observation of nematode movement toward or away from a test compound. The fundamental differences in the two assay setups (measuring tracks versus direct nematode observation) may explain the differences in observed results. Our results found $H$. glycines $\mathrm{J} 2$ s to be strongly attracted to both $\mathrm{KNO}_{3}$ and $\mathrm{NH}_{4} \mathrm{NO}_{3}$ and repelled by $\mathrm{CaCl}_{2}$, which has not been reported previously. As shown in Figure 2B, the attraction of $H$. glycines to $\mathrm{KNO}_{3}$ occurred within a few hours, with a large number of $\mathbf{J} 2 \mathrm{~s}$ being attracted to the chemical as early as $4 \mathrm{~h}$ after the start of the experiment. This response indicates that a $\mathrm{KNO}_{3}$ solution can serve as a good positive control for future $\mathrm{H}$. glycines chemotaxis studies.

The root chip system provides an alternative methodology for experiments that test nematode behavior in response to collected root exudates. Previous work has relied on collecting root exudates from growing plants and applied them to nematodes in separate steps (Levene et al. 1998; Tefft and Bone 1985; Zhao et al. 2000). Our root chip allows for undisturbed growth of the soybean seedlings in the presence of the nematode. Therefore, the nematode can react to exudates as they are directly being emitted from the roots. A single chip has four separate lanes that allow spatial effects of roots of plant-parasitic nematode chemotaxis to be discerned. Spatial preference of $\mathbf{J} 2$ for penetration has been noted for species such as $M$. incognita, which migrates to root tips of host plants for penetration (Wyss et al. 1992). The results of our experiments with 3 -day-old soybean seedlings suggest there is no spatial preference on chemotaxis of $H$. glycines (Table 2 ).

Experiments with the root chip were conducted by taking highresolution scans of the chips under controlled conditions over a period of $18 \mathrm{~h}$. The $H$. glycines $\mathrm{J} 2 \mathrm{~s}$ tested with the root chip were successfully monitored and analyzed with custom software program for automatic image capture as well as to streamline image processing and analysis. Results from the root chip found $H$. glycines $\mathrm{J} 2$ s to be attracted to roots of susceptible (Williams 82) soybean across multiple runs. The $H$. glycines $\mathbf{J} 2$ s were attracted to resistant (Jack) soybean in some runs (Fig. 4), but this effect was not consistent across runs (Fig. 5). There was no attraction of $H$. glycines $\mathrm{J} 2 \mathrm{~s}$ toward susceptible or resistant roots when both were placed on opposite sides of the chip (Fig. 5). This result is not inconsistent with the findings of Colgrove and Niblack (2005) who reported no differences in $H$. glycines $\mathrm{J} 2$ penetration into roots of resistant and susceptible soybean genotypes. Our results do not provide any strong conclusions in understanding the role of plant resistance on $H$. glycines chemotaxis, because the two soybean cultivars tested are genetically distinct. Because we did not test the effect of roots of nonhosts, it also is unclear if the attraction of $H$. glycines to the roots in our experiments is soybean specific or due to exudates found in all plant roots. Others have reported host and cultivar effects on chemotaxis of plant-parasitic nematodes (Zhao et al. 2000), indicating that this assay might be useful for further host and cultivar preference studies. Additionally, this assay may be valuable for testing the effect of seed treatments, particularly systemic compounds, on chemotaxis of different nematode species.

Recently, Hida et al. (2015) described a microfluidic chip that measured chemotaxis of $M$. incognita $\mathrm{J} 2 \mathrm{~s}$ to $\mathrm{KNO}_{3}$. There are similarities and differences between their approaches and ours. Both chips were constructed from PDMS using soft lithography, incorporate microscale filters to form concentration gradients of a chemical, and require the $\mathrm{J} 2 \mathrm{~s}$ to migrate to a final resting chamber. The chip described by Hida et al. (2015) is a T-shaped design that requires the nematodes to migrate up a channel before making a choice toward or away from a test chemical. Our chips require the $\mathrm{J} 2 \mathrm{~s}$ to make an immediate choice toward or away from a treatment.
Additionally, Hida et al. (2015) used agarose gel as the migration medium, and our chips were filled with sterile, distilled water. The chips described in Hida et al. (2015) as well as in our paper can aid in understanding plant-parasitic nematode chemotaxis.

There are some limitations of the microfluidic chips presented in this paper. As with other in vitro assays, it is difficult to ascertain if the results obtained from laboratory assays can be reliably reproduced in the field setting. For instance, the chemotactic gradients established in our assays may be different from those in natural soil environment, since these gradients may be altered by abiotic or biotic factors in the soil. In this context, Spence et al. (2008) cautioned that while laboratory assays can be useful in studying plant-parasitic and entomopathogenic nematodes, the effects observed in an in vitro assay can only provide predictions of field-relevant interactions and extrapolations of laboratory results are often unjustified. Advances in replicating the soil environment within chemotactic assays may aid in providing better predictions of field-relevant interactions (Lockery et al. 2008). Additionally, microfluidic chips constructed from PDMS are unable to test the chemotaxic effects of hydrophobic compounds without additional fabrication steps (Mukhopadhyay 2007; Toepke and Beebe 2006). Our chips rely on diffusion of compounds in water, and hydrophobic compounds would be immiscible and tend to get absorbed into the PDMS sidewalls. In order to use PDMS microfluidic chips with hydrophobic compounds, the chips must be coated with a suitable hydrophobic coating (e.g., Teflon, paralyne) to prevent the hydrophobic compound from being absorbed into the PDMS material.

Microfluidic technology offers an attractive alternative to agarbased plate assays for studying chemotaxis of plant-parasitic nematodes. Our results demonstrate the direct benefits of microfluidic chips for testing a large number of nematodes, creating a controlled chemical gradient for long time periods, running concurrent, parallel experiments on the same chip, monitoring root-nematode interactions, and using semiautomated imaging and tracking tools during the experiments. The use of microfluidic chips paired with image capture tools can provide a wealth of information about nematode behavior. Increased adoption, improvements, and modifications of microfluidic chips to answer specific research questions may result in greater data granularity and faster screening of the chemotactic effects of chemicals and root exudates on plant-parasitic nematodes.

\section{ACKNOWLEDGMENTS}

This work was partially supported by funding from the National Science Foundation CBET-1150867 to S. Pandey. This material is based upon work supported by the National Science Foundation I/UCRC, the Center for Arthropod Management Technologies under Grant No. IIP-1338775 and by industry partners. We are grateful for the support and advice provided by B. Bonning (Iowa State University) and the Center's advisory board members, and to J. Jensen for assistance in research and manuscript preparation.

\section{LITERATURE CITED}

Beebe, D. J., Mensing, G. A., and Walker, G. M. 2002. Physics and applications of microfluidics in biology. Annu. Rev. Biomed. Eng. 4:261-286.

Carr, J. A., Parashar, A., Gibson, R., Robertson, A. P., Martin, R. J., and Pandey, S. 2011. A microfluidic platform for high-sensitivity, real-time drug screening on C. elegans and parasitic nematodes. Lab Chip 11:2385-2396.

Castro, C. E., Belser, N. O., McKinney, H. E., and Thomason, I. J. 1990. Strong repellency of the root knot nematode, Meloidogyne incognita by specific inorganic ions. J. Chem. Ecol. 16:1199-1205.

Chen, B., Deutmeyer, A., Carr, J., Roberts, A. P., Martin, R. J., and Pandey, S. 2011. Microfluidic bioassay to characterize parasitic nematode phenotype and antihelminthic resistance. Parasitology 138:80-88.

Colgrove, A. L., and Niblack, T. L. 2005. The effect of resistant soybean on male and female development and adult sex ratios of Heterodera glycines. J. Nematol. 37:161-167.

Curtis, R. H. C. 2008. Plant-nematode interactions: Environmental signals detected by the nematode's chemosensory organs control changes in the surface cuticle and behaviour. Parasite 15:310-316. 
Devine, K. J., and Jones, P. W. 2003. Investigations into the chemo-attraction of the potato cyst nematodes Globodera rostochiensis and G. pallida towards fractionated potato leachate. Nematology 5:65-75.

Dusenbery, D. B. 1983. Chemotactic behavior of nematodes. J. Nematol. 15: 168-173.

Faghihi, J., and Ferris, J. M. 2000. An efficient new device to release eggs from Heterodera glycines. J. Nematol. 32:411-413.

Hida, H., Nishiyama, H., Sawa, S., Higashiyama, T., and Arata, H. 2015. Chemotaxis assay of plant-parasitic nematodes on a gel-filled microchannel device. Sens. Actuat. B 221:1483-1491.

Hiltpold, I., Jaffuel, G., and Turlings, T. C. J. 2014. The dual effects of root-cap exudates on nematodes: from quiescence in plant-parasitic nematodes to frenzy in entomopathogenic nematodes. J. Exp. Bot. 66: 421-424.

Hu, Y., Zhang, W., Zhang, P., Ruan, W., and Zhu, X. 2013. Nematicidal activity of chaetoglobosin A produced by Chaetomium globosom NK102 against Meloidogyne incognita. J. Agric. Food Chem. 61:41-46.

Huettel, R. N., and Jaffe, H. 1987. Attraction and behavior of Heterodera glycines, the soybean cyst nematode, to some biological and inorganic compounds. Proc. Helminthol. Soc. Wash. 54:122-125.

Hussey, R. S., and Barker, K. R. 1973. A comparison of methods of collecting inocula of Meloidogyne spp., including a new technique. Plant Dis. Rep. 57: 1025-1028.

Jenkins, W. R. 1964. A rapid centrifugal-flotation technique for separating nematodes from soil. Plant Dis. Rep. 48:692.

Kerry, B. R. 2000. Rhizosphere interactions and the exploitation of microbial agents for the biological control of plant-parasitic nematodes. Annu. Rev. Phytopathol. 38:423-441.

Le Saux, R., and Quénéhervé, P. 2002. Differential chemotactic responses of two plant-parasitic nematodes, Meloidogyne incognita and Rotylenchulus reniformis, to some inorganic ions. Nematology 4:99-105.

Levene, B. C., Owen, M. D., and Tylka, G. L. 1998. Influence of herbicide application to soybeans on soybean cyst nematode egg hatching. J. Nematol. 30:347-352.

Linsell, K. J., Riley, I. T., Davies, K. A., and Oldach, K. H. 2014. Characterization of resistance to Pratylenchus thornei (Nematoda) in wheat (Triticum aestivum): Attraction, penetration, motility, and reproduction. Phytopathology 104:174-187.

Liu, B., Hibbard, J. K., Urwin, P. E., and Atkinson, H. W. 2005. The production of synthetic chemodisruptive peptides in planta disrupts the establishment of cyst nematodes. Plant Biotechnol. J. 3:487-496.

Lockery, S. R., Hulme, S. E., Roberts, W. M., Robinson, K. J., Laromaine, A., Lindsay, T. H., Whitesides, G. M., and Weeks, J. C. 2012. A microfluidic device for whole-animal drug screening using electrophysiological measures in the nematode C. elegans. Lab Chip 12:2211-2220.

Lockery, S. R., Lawton, K. J., Doll, J. C., Faumont, S., Coulthard, S. M., Thiele, T. R., Chronis, N., McCormick, K. E., Goodman, M. B., and Pruitt, B. L. 2008. Artificial dirt: Microfluidic substrate for nematode neurobiology and behavior. J. Neurophysiol. 99:3136-3143.

Mukhopadhyay, R. 2007. When PDMS isn't the best. What are its weaknesses, and which other polymers can researchers add to their toolboxes? Anal. Chem. 79:3249-3253.

Njus, Z., Feldmann, D., Brien, R., Kong, T., Kalwa, U., and Pandey, S. 2015. Characterizing the effect of static magnetic fields on $C$. elegans using microfluidics. Adv. Biosci. Biotechnol. 6:583-591.
Papademetriou, M. K., and Bone, L. W. 1983. Chemotaxis of larval soybean cyst nematode, Heterodera glycines race 3 , to root leachates and ions. J. Chem. Ecol. 9:387-396.

Perry, R. N. 1996. Chemoreception in plant-parasitic nematodes. Annu. Rev. Phytopathol. 34:181-199.

Peytavi, R., Raymond, F. R., Gagné, D., Picard, F. J., Jia, G., Zoval, J., Madou, M., Boissinot, K., Boissinot, M., Bissonnette, L., Ouellette, M., and Bergeron, M. G. 2005. Microfluidic device for rapid (<15 min) automated microarray hybridization. Clin. Chem. 51:1836-1844.

Prot, J. C. 1979. Influence of concentration gradients of salts on the behaviour of four plant parasitic nematodes. Rev. Nematologie 2:11-16.

Reynolds, A. M., Dutta, T. K., Curtis, R. H. C., Powers, S. J., Gaur, H. G., and Kerry, B. R. 2011. Chemotaxis can take plant-parasitic nematodes to the source of a chemo-attractant via the shortest possible routes. J. R. Soc. Interface 8:568-577.

Riddle, D. L., and Bird, A. F. 1985. Responses of the plant parasitic nematodes Rotylenchulus reniformis, Anguina agrostis and Meloidogyne javanica to chemical attractants. Parasitology 91:185-195.

Saldanha, J. N., Parashar, A., Pandey, S., and Powell-Coffmann, J. A. 2013. Multiparameter behavioral analyses provide insights into mechanisms of cyanide resistance in Caenorhabditis elegans. Toxicol. Sci. 135:156-168.

Sasser, J. N., and Freckman, D. W. 1987. A world perspective on nematology: The role of the society. Pages 7-14 in: Vistas on Nematology. J. A. Veeck and D. W. Dickson, eds. Society of Nematologists, Hyattsville, MD.

Shinya, R., Chen, A., and Sternberg, P. W. 2015. Attraction and mating in Bursaphelenchus okinawaensis and B. xylophilus. J. Nematol. 47:176-183.

Sikora, R. A. 1992. Management of the antagonistic potential in agricultural ecosystems for the biological control of plant parasitic nematode. Annu. Rev. Phytopathol. 30:245-270.

Spence, K. O., Lewis, E. E., and Perry, R. N. 2008. Host-finding and invasion by entomopathogenic and plant-parasitic nematodes: Evaluating the ability of laboratory bioassays to predict field results. J. Nematol. 40:93-98.

Tefft, P. M., and Bone, L. W. 1985. Plant-induced hatching of eggs of the soybean cyst nematode Heterodera glycines. J. Nematol. 17:275-279.

Toepke, M. W., and Beebe, D. J. 2006. PDMS absorption of small molecules and consequences in microfluidic application. Lab Chip 6:1484-1486.

Wang, C., Bruening, G., and Williamson, V. M. 2009a. Determination of preferred $\mathrm{pH}$ for root-knot nematode aggregation using pluronic F-127 gel. J. Chem. Ecol. 35:1242-1251.

Wang, C., Lower, S., and Williamson, V. M. 2009b. Application of Pluronic gel to the study of root-knot nematode behavior. Nematology 11:453-464.

Wong, A. T., Tylka, G. L., and Hartzler, R. G. 1993. Effects of eight herbicides on in vitro hatching of Heterodera glycines. J. Nematol. 25:578-584.

Wuyts, N., Swennen, R., and de Waele, D. 2006. Effects of plant phenylpropanoid pathway products and selected terpenoids and alkaloids on the behaviour of the plant-parasitic nematodes Radopholus similis, Pratylenchus penetrans, and Meloidogyne incognita. Nematology 8:89-101.

Wyss, U., Grundler, F. M. W., and Munch, A. 1992. The parasitic behaviour of second-stage juveniles of Meloidogyne incognita in roots of Arabidopsis thaliana. Nematologica 38:98-111.

Zhao, X., Schmitt, M., and Hawes, M. C. 2000. Species-dependent effect of border cell and root tip exudates on nematode behavior. Phytopathology 90 : 1239-1245.

Zuckerman, B. M., and Jansson, H. 1984. Nematode chemotaxis and possible mechanisms of host/prey recognition. Annu. Rev. Phytopathol. 22:95-113. 